PROCEEDINGS OF THE

AMERICAN MATHEMATICAL SOCIETY

Volume 134, Number 7, Pages 2161-2163

S 0002-9939(06)08389-4

Article electronically published on January 17, 2006

\title{
THE PRODUCT OF A BAIRE SPACE WITH A HEREDITARILY BAIRE METRIC SPACE IS BAIRE
}

\author{
WARREN B. MOORS
}

(Communicated by Jonathan M. Borwein)

\begin{abstract}
In this paper we prove that the product of a Baire space with a metrizable hereditarily Baire space is again a Baire space. This answers a recent question of J. Chaber and R. Pol.
\end{abstract}

A topological space $X$ is called a Baire space if for each sequence $\left(O_{n}: n \in \mathbb{N}\right)$ of dense open subsets of $X, \bigcap_{n \in \mathbb{N}} O_{n}$ is dense in $X$, and a Baire space $Y$ is called barely Baire if there exists a Baire space $Z$ such that $Y \times Z$ is not Baire. It is well known that there exist metrizable barely Baire spaces (see 2]). On the other hand, it has recently been shown in [1] that the arbitrary product of hereditarily Baire (i.e., each closed subspace of them is Baire) metrizable spaces is again Baire. However, in this same paper the authors lament that they do not know whether there exists a metrizable Baire space $X$ and a metrizable hereditarily Baire space $Y$ such that $X \times Y$ is not Baire. In this paper we resolve this situation. Specifically, we show that if $X$ is Baire and $Y$ is hereditarily Baire and metrizable, then $X \times Y$ is also Baire. In order to simplify the appearance of the proof of our main theorem we shall invoke the machinery of topological games. In particular, we shall use the game characterisation of Baireness due to Saint Raymond, [3].

Let $(X, \tau)$ be a topological space. On $X$ we consider the Choquet game $\mathcal{G}_{X}$ played between two players $\alpha$ and $\beta$. The player $\beta$ goes first (always!) and chooses a non-empty open subset $B_{1} \subseteq X$. Player $\alpha$ must then respond by choosing a non-empty open subset $A_{1} \subseteq B_{1}$. Following this, player $\beta$ must select another non-empty open subset $B_{2} \subseteq A_{1} \subseteq B_{1}$ and in turn player $\alpha$ must again respond by selecting a non-empty open subset $A_{2} \subseteq B_{2} \subseteq A_{1} \subseteq B_{1}$. Continuing this procedure indefinitely the players $\alpha$ and $\beta$ produce a sequence $\left(\left(A_{n}, B_{n}\right): n \in \mathbb{N}\right)$ of pairs of open subsets called a play of the $\mathcal{G}_{X}$-game. We shall declare that $\alpha$ wins a play $\left(\left(A_{n}, B_{n}\right): n \in \mathbb{N}\right)$ of the $\mathcal{G}_{X}$-game if $\bigcap_{n \in \mathbb{N}} A_{n} \neq \emptyset$; otherwise the player $\beta$ is said to have won. By a strategy $t$ for the player $\beta$ we mean a "rule" that specifies each move of the player $\beta$ in every possible situation that can occur. More precisely, a strategy $t:=\left(t_{n}: n \in \mathbb{N}\right)$ for $\beta$ is a sequence of $\tau$-valued functions such that $\emptyset \neq t_{n+1}\left(A_{1}, \ldots, A_{n}\right) \subseteq A_{n}$ for each $n \in \mathbb{N}$. The domain of each function $t_{n}$ is precisely the set of all finite sequences $\left(A_{1}, \ldots, A_{n-1}\right)$ of length $n-1$ in $\tau \backslash\{\emptyset\}$ with

Received by the editors February 18, 2005.

2000 Mathematics Subject Classification. Primary 54B10, 54C35; Secondary 54E52.

Key words and phrases. Baire space, hereditarily Baire space, product space.

The author was supported by the Marsden Fund research grant, UOA0422, administered by the Royal Society of New Zealand. 
$A_{j} \subseteq t_{j}\left(A_{1}, \ldots, A_{j-1}\right)$ for all $1 \leq j \leq n-1$. (Note: the sequence of length 0 will be denoted by $\emptyset$.) Such a finite sequence $\left(A_{1}, A_{2}, \ldots, A_{n-1}\right)$ or infinite sequence $\left(A_{n}: n \in \mathbb{N}\right)$ is called a $t$-sequence. A strategy $t:=\left(t_{n}: n \in \mathbb{N}\right)$ for the player $\beta$ is called a winning strategy if each infinite $t$-sequence is won by $\beta$.

Theorem 1 ([3]). Let $X$ be a topological space. Then $X$ is a Baire space if, and only if, $\beta$ does not have a winning strategy in the Choquet game played on $X$.

The following result is proved by a straightforward induction (on the number of elements of $Z$ ) argument.

Lemma 1. Let $X$ be a topological space, let $(Y, d)$ be a metric space and let $O$ be a dense open subset of $X \times Y$. Then given any finite subset $Z$ of $Y, \varepsilon>0$ and nonempty open subset $U$ of $X$ there exists a finite subset $Y^{\prime}$ of $Y$ and a non-empty open subset $V$ of $U$ such that: (i) for each $z \in Z$ there exists a $y \in Y^{\prime}$ with $d(y, z)<\varepsilon$ and (ii) $V \times Y^{\prime} \subseteq O$.

Theorem 2. Let $X$ be a Baire space and let $(Y, d)$ be a hereditarily Baire metric space. Then $X \times Y$ is a Baire space.

Proof. Let $\left(O_{n}: n \in \mathbb{N}\right)$ be a decreasing sequence of dense open subsets of $X \times Y$. We need to show that $\bigcap_{n \in \mathbb{N}} O_{n}$ is dense in $X \times Y$. To this end, let $U$ be a nonempty open subset of $X$ and let $V$ be a non-empty open subset of $Y$; we will show that $\left(\bigcap_{n \in \mathbb{N}} O_{n}\right) \cap(U \times V) \neq \emptyset$. To achieve this we will appeal to the game characterisation of Baireness given in Theorem 1. Thus, we shall inductively define a (necessarily non-winning) strategy $t:=\left(t_{n}: n \in \mathbb{N}\right)$ for the player $\beta$ in the Choquet game played on $X$.

Step 1 . Choose $y \in V$ and a non-empty open subset $U_{\emptyset} \subseteq U$ such that $U_{\emptyset} \times\{y\} \subseteq$ $O_{1}$. Note this choice is possible since $(U \times V) \cap O_{1} \neq \emptyset$. Let $Y_{\emptyset}:=\{y\}, Z_{\emptyset}:=Y_{\emptyset}$ and define $t_{1}(\emptyset):=U_{\emptyset}$.

Step 2. For each $t$-sequence $\left(A_{1}\right)$ of length 1 we can apply Lemma 1 to get a finite subset $Y_{\left(A_{1}\right)}$ of $V$ and a non-empty open subset $U_{\left(A_{1}\right)}$ of $A_{1}$ so that:

(i) for each $z \in Z_{\emptyset}$ there exists a $y \in Y_{\left(A_{1}\right)}$ such that $d(y, z)<1 / 2$;

(ii) $U_{\left(A_{1}\right)} \times Y_{\left(A_{1}\right)} \subseteq O_{2}$.

Then we define

(iii) $Z_{\left(A_{1}\right)}:=Z_{\emptyset} \cup Y_{\left(A_{1}\right)}$;

(iv) $t_{2}\left(A_{1}\right):=U_{\left(A_{1}\right)}$.

Now suppose that the finite subsets $Y_{\left(A_{1}, \ldots, A_{j}\right)}$ and $Z_{\left(A_{1}, \ldots, A_{j}\right)}$ of $V$, the nonempty open subset $U_{\left(A_{1}, \ldots, A_{j}\right)}$ of $A_{j}$ and the strategy $t_{j+1}$ have been defined for each $t$-sequence $\left(A_{1}, \ldots, A_{j}\right)$ of length $j$ with $1 \leq j \leq(n-1)$ and $2 \leq n \in \mathbb{N}$ so that:

(i) for each $z \in Z_{\left(A_{1}, \ldots, A_{j-1}\right)}$ there exists a $y \in Y_{\left(A_{1}, \ldots, A_{j}\right)}$ such that $d(y, z)<$ $1 /(j+1)$

(ii) $U_{\left(A_{1}, \ldots, A_{j}\right)} \times Y_{\left(A_{1}, \ldots, A_{j}\right)} \subseteq O_{j+1}$;

(iii) $Z_{\left(A_{1}, \ldots, A_{j}\right)}:=Z_{\left(A_{1}, \ldots, A_{j-1}\right)} \cup Y_{\left(A_{1}, \ldots, A_{j}\right)}$;

(iv) $t_{j+1}\left(A_{1}, \ldots, A_{j}\right):=U_{\left(A_{1}, \ldots, A_{j}\right)}$.

Step $(n+1)$. For each $t$-sequence $\left(A_{1}, \ldots, A_{n}\right)$ of length $n$ we can apply Lemma 1 to get a finite subset $Y_{\left(A_{1}, \ldots, A_{n}\right)}$ of $V$ and a non-empty open subset $U_{\left(A_{1}, \ldots, A_{n}\right)}$ of 
$A_{n}$ so that:

(i) for each $z \in Z_{\left(A_{1}, \ldots, A_{n-1}\right)}$ there exists a $y \in Y_{\left(A_{1}, \ldots, A_{n}\right)}$ such that $d(y, z)<$ $1 /(n+1)$

(ii) $U_{\left(A_{1}, \ldots, A_{n}\right)} \times Y_{\left(A_{1}, \ldots, A_{n}\right)} \subseteq O_{n+1}$.

Then we define

(iii) $Z_{\left(A_{1}, \ldots, A_{n}\right)}:=Z_{\left(A_{1}, \ldots, A_{n-1}\right)} \cup Y_{\left(A_{1}, \ldots, A_{n}\right)}$;

(iv) $t_{n+1}\left(A_{1}, \ldots, A_{n}\right):=U_{\left(A_{1}, \ldots, A_{n}\right)}$.

This completes the definition of $t:=\left(t_{n}: n \in \mathbb{N}\right)$. Now since $X$ is a Baire space $t$ is not a winning strategy for the player $\beta$ in the $\mathcal{G}_{X}$-game. Therefore there exists a $t$-sequence $\left(A_{n}: n \in \mathbb{N}\right)$ where $\bigcap_{n \in \mathbb{N}} A_{n} \neq \emptyset$ (i.e., $\alpha$ wins). Choose $x \in \bigcap_{n \in \mathbb{N}} A_{n}$ and define, for each $n \in \mathbb{N}$, the open sets $\left(W_{n}: n \in \mathbb{N}\right)$ of $Y$ so that $\{x\} \times W_{n}:=(\{x\} \times Y) \cap O_{n}$.

Let $Z:=\bigcup\left\{Z_{\left(A_{1}, \ldots, A_{n-1}\right)}: n \in \mathbb{N}\right\} \subseteq V$. Then $\bar{Z}$ is a Baire space and by construction $W_{n} \cap Z$ is dense in $Z$ for each $n \in \mathbb{N}$. Indeed, if $z \in Z, n \in \mathbb{N}$ and $\varepsilon>0$, then we can choose $k \in \mathbb{N}$ sufficiently large so that $n<k, 1 / k<\varepsilon$ and $z \in Z_{\left(A_{1}, \ldots, A_{k-1}\right)}$. Then there exists a $y \in Y_{\left(A_{1}, \ldots, A_{k}\right)}$ such that $d(y, z)<$ $1 /(k+1)<\varepsilon$ and $(x, y) \in O_{k+1} \cap(\{x\} \times Y)$, which implies that $y \in W_{k+1} \subseteq W_{n}$. Thus, $y \in B(z, \varepsilon) \cap\left(W_{n} \cap Z\right) \neq \emptyset$. Next, choose $y \in \bigcap_{n \in \mathbb{N}} W_{n} \cap V \cap \bar{Z}$. Then $(x, y) \in\left(\bigcap_{n \in \mathbb{N}} O_{n}\right) \cap(U \times V) \neq \emptyset$, which completes the proof.

Remark. In the previous theorem it is possible to weaken the hypothesis on $Y$ while not affecting the conclusion (i.e., that $X \times Y$ is Baire). For example, if $Y$ is hereditarily Baire and first countable, then it is not difficult to see how to modify the proof in order to retain the same conclusion. If one is willing to invest a bit more effort, then it can be shown that if $Y$ is expressible as a product of hereditarily Baire metric spaces, then $X \times Y$ is Baire, despite the fact, that in this particular case, $Y$ is not necessarily obliged to be hereditarily Baire (see 1 for the idea behind this).

\section{REFERENCES}

[1] J. Chaber, R. Pol, On hereditarily Baire spaces, $\sigma$-fragmentability of mappings and Namioka property, Topology Appl. 151 (2005), 132-143. MR.2139747

[2] W. G. Fleissner, K. Kunen, Barely Baire spaces, Fund. Math. 101 (1978), 229-240. MR0521125 (80f:54009)

[3] J. Saint Raymond, Jeux topologiques et espaces de Namioka, Proc. Amer. Math. Soc. 87 (1983), 499-504. MR0684646 (83m:54060)

Department of Mathematics, The University of Auckland, Private Bag 92019, AuckLAND, NEW ZEALAND

E-mail address: moors@math.auckland.ac.nz 